when non-surgical treatment was selected, the omission of brachytherapy should be avoided.

Disclosures The authors declare that they have no competing interests.

\section{REAL-WORLD HEALTHCARE COSTS BY LINE OF THERAPY AMONG NEWLY DIAGNOSED CERVICAL CANCER PATIENTS AND CERVICAL CANCER PATIENTS NEWLY INITIATING SYSTEMIC THERAPIES}

${ }^{1}$ Nehemiah Kebede, ${ }^{1}$ Ruchitbhai Shah, ${ }^{1}$ Anuj Shah, ${ }^{2}$ Shelby Corman, ${ }^{3}$ Chizoba Nwankwo. ${ }^{1}$ Pharmerit - an Open Health Company; Pharmerit International, Bethesda, MD, USA; ${ }^{2}$ Pharmerit - an Open Health Company; USA; ${ }^{3}$ Merck and Co., Inc

\subsection{6/ijgc-2020-ESG0.29}

Introduction/Background There are little data describing the economic burden among newly diagnosed and advanced cervical cancer patients, by line of therapy (L). NCCN recommends the use of systemic treatment for advanced cervical cancer patients. Therefore, this study aimed to assess healthcare costs among newly diagnosed cervical cancer patients and those newly initiating systemic treatments.

Methodology This was a retrospective observational study conducted using the Optum Clinformatics DataMart database. The first cohort consisted of cervical cancer patients newly diagnosed between January 2015 - June 2018, and continuous enrollment for 12 months prior and 6 months post diagnosis. The start of $1 \mathrm{~L}$ was the date of the first treatment. Treatments initiated within 90 days of a surgery or the end of radiotherapy, and systemic treatment started within 28 days of any previous treatment were part of the same treatment line.

The second cohort consisted of cervical cancer patients with $\geq 2$ claims for systemic therapy (i.e., chemo- or immunotherapy) within a 4-week period between June 2014 - October 2018, and continuously enrolled for 6 months prior and 3 months post therapy initiation. All claims for the same systemic therapy without a $>90$-day gap, or initiation of a new systemic therapy within 28 days of a previous treatment were attributed to the same treatment line. Claims for adjuvant systemic therapy (i.e., within \pm 90 -days of a cervical cancerrelated surgery) were excluded.

The per patient per month (PPPM) components of healthcare costs attributable to $1 \mathrm{~L}$ and $2 \mathrm{~L}$ were summarized for both cohorts (figures 1 and 2). Analyses for the second cohort were stratified by the presence of comorbid non-cervical cancers prior to systemic therapy initiation.

Results The first cohort included 655 patients who received at least $1 \mathrm{~L}$ of which 162 received $2 \mathrm{~L}$. The mean PPPM healthcare cost from diagnosis to end of follow-up was $\$ 10,121$. The mean PPPM healthcare costs (figure 1) attributable to $2 \mathrm{~L}$ $(\$ 15,183)$ exceeded that of $1 \mathrm{~L}(\$ 10,929)$.

The second cohort included 1,229 patients who newly initiated $1 \mathrm{~L}$ of which 357 received $2 \mathrm{~L}$. The mean PPPM healthcare cost from initiation of systemic therapy to end of followup was $\$ 15,463$. The PPPM healthcare costs (figure 2) was higher during 2L versus 1L (without prior cancers: \$22,973 vs $\$ 13,044$; with prior cancers: $\$ 19,822$ vs $\$ 16,387)$. Outpatient costs accounted for $>70 \%$ of total PPPM healthcare costs attributable to $1 \mathrm{~L}$ and $2 \mathrm{~L}$ for both cohorts.

Conclusion Moving from $1 \mathrm{~L}$ to $2 \mathrm{~L}$ was associated with an increase in healthcare costs which may be indicative of disease progression/recurrence.

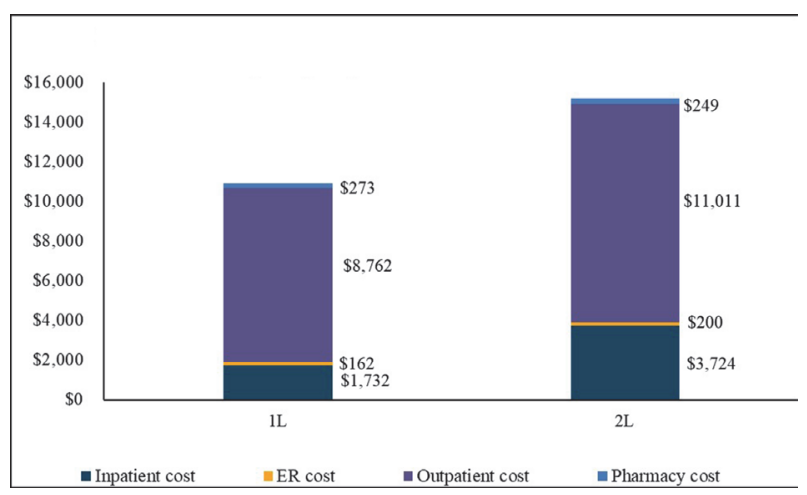

Abstract 361 Figure 1 Mean per patient per month healthcare costs by line of therapy among newly diagnosed cervical cancer patients

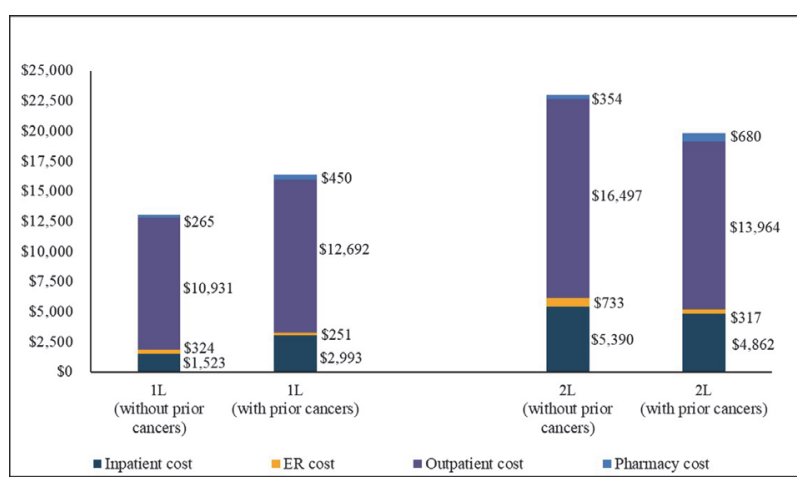

Abstract 361 Figure 2 Mean per patient per month healthcare costs by line of therapy among cervical cancer patients newly initiating systemic therapy

Disclosures This study was funded by Merck Sharp \& Dohme Corp., a subsidiary of Merck \& Co., Inc., Kenilworth, NJ, USA. Chizoba Nwankwo is an employee of Merck Sharp \& Dohme Corp., a subsidiary of Merck \& Co., Inc., Kenilworth, NJ, USA. Anuj Shah, Ruchit Shah, Shelby Corman, and Nehemiah Kebede are employees of Pharmerit International, which received consulting fees related to this study.

\section{STUDY OF NEOADJUVANT CHEMOTHERAPY WITH A CARBOPLATIN AND TAXOL FOLLOWED BY CHEMORADIATION VERSUS CHEMORADIATION FOR LOCALLY ADVANCED CERVICAL CANCER}

${ }^{1}$ Hanady Hegazy, ${ }^{1}$ Neamat Hegazy, ${ }^{1}$ Maher Soliman, ${ }^{2}$ Amr Elsaid. ${ }^{1}$ Alexandria Faculty of Medicine; Clinical Oncology; ${ }^{2}$ Alexandria Faculty of Medicine

\subsection{6/ijgc-2020-ESG0.30}

Introduction/Background The incidence of locally advanced cancer cervix (LACC) in developing countries is still high due to the absence of regular screening programs and vaccination. Besides, the survival in LACC patients remains low. So we conducted this study to evaluate the use of neoadjuvant chemotherapy (NAC) induction before the definitive concomitant chemoradiation (CCR) for downstaging of the tumor.

Methodology A 68 patients with LACC (International Federation of Gynecology and Obstetrics FIGO stage IB2 to IVA) randomly assigned to group A (received three cycles of NAC with Carboplatin AUC5 + Taxol $175 \mathrm{mg} / \mathrm{m} 2$ followed by the 
standard CCR with weekly Cisplatin plus 3D conformal pelvic radiotherapy followed by brachytherapy (BT)) and group B ( received the standard CCR and $\mathrm{BT}$ ). The primary end point: the assessment of response rate and local control. The secondary end points: assessment of the 2 years overall survival (OS) and progression free survival (PFS).

Results The median age was 54 years old and a range of 44 years old with a performance status $(0,1)$. The majority of patients had squamous cell carcinoma $(88.24 \%$ group A vs. $94.12 \%$ group $\mathrm{B}$ ) and most of the patients were FIGO stage IIIC, IVA and IIB (41.18\%, 32.35\% and $20.59 \%$ in group A vs. $32.35 \%, 17.65 \%$ and $17.65 \%$ in group B respectively). CT abdomen and pelvis done at time of diagnosis showed pathological enlarged lymph nodes in $64.71 \%$ and $58.82 \%$ of patients in group A and B respectively. After NAC, $97.06 \%$ of the patients achieved partial response with a reduction of tumor volume by $76.07 \%$ and only $2.94 \%$ had stable disease. Higher partial response in groups A $(55.88 \%$ in group A vs. $32.35 \%$ in group B, p value 0.151 ) and higher overall response rate (ORR) in group A $(79.41 \%$ vs.70.59\%) while local control was higher in group B $(91.18 \%$ vs. $97.06 \%$, p value 0.614). The 2 years PFS was $91 \%$ in group $A$ and $97.1 \%$ in group B and OS of $100 \%$ as all the patients remain alive till the end of the 2 years follow up.

Conclusion The addition of NAC to the standard CCR achieved a higher partial response rate and ORR with a reasonable local control of the disease. This can facilitate the CCR plane and subsequently the brachytherapy planning parameters in locally advanced cases with no inferiority of the PFS and OS compared to the standard.

Disclosures The authors of this abstract do not have any research support and no conflict of interest.

\section{MUCOADHESIVE BILAYER VAGINAL TABLET AS A POTENTIAL ADJUVANT TREATMENT FOR CERVICAL CANCER}

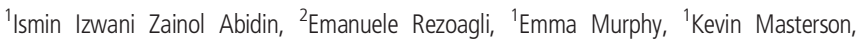
${ }^{1}$ Neil Rowan, ${ }^{1}$ lan Major. ${ }^{1}$ Athlone Institute of Technology; Bioscience Research Institute; ${ }^{2}$ Department of Medicine and Surgery, University of Milano-Bicocca, Monza, Italy; Lung Biology Group, Regenerative Medicine Institute at Curam Centre for Medical Devices, National University of Ireland Galway, Ireland; Bioscience Research Institute Athlone

\subsection{6/ijgc-2020-ESG0.31}

Introduction/Background Cervical cancer (CC) is described as cancer that occurs in the cells located in the cervix. Each year, more than 500,000 women are diagnosed with CC and the disease results in over 300,000 deaths across the world. Infection by high-risk oncogenic subtypes of human papillomavirus (HPV); HPV16 and HPV18, is the cause for almost all cases of CC. In developed countries, CC incidence and mortality have more than halved over the past 30 years as they were able to establish successful national HPV screening and vaccination programs. However, this disease remains persistent and has become one of the leading causes of death among women in developing (low to middle-income) countries; mostly in African countries. Adapting to low resources, developing countries have practiced a feasible and cost-effective solutions with the screen and treat approach instead. Screening usually involves visual inspection with acetic acid (VIA) or Lugol's iodine (VILI) that gives immediate results, facilitating instantaneous treatment strategies and prevents loss to followup. Treatments includes loop electrosurgical excision procedure
(LEEP), cryotherapy or cold-knife conization. This research proposes the development of a self-administered chemotherapeutic vaginal tablet formulation that would complement these current treatment strategies.

Methodology The bilayer tablets were prepared one layer at a time, using a single punch direct compression machine. Tablets were then evaluated using the pharmacopeial guideline that include tablet uniformity, hardness, friability and content uniformity. Swelling test was performed by simple immersion of the tablets in dissolution medium for 24 hours, the tablet's weight before and after were recorded. The drug release profile was evaluated by in vitro drug dissolution test using the USP paddle method in $2 \%$ aqueous sodium dodecyl sulphate (SDS) solution; maintained at $100 \mathrm{rpm}, 37 \pm 1^{\circ} \mathrm{C}$ and in sink conditions. All samples were measured spectroscopically. Cell viability after treatment with the drugs was determined using the MTT assay on Ca-ski cells.

Results Results showed that the tablet of this combination are uniform and durable in compliance to pharmacopeial standards, a swelling study shows promising potential for mucoadhesion and has an extended in vitro drug release profile over 72 hours. In vitro cell culture with Ca-Ski cells however, did not show a synergistic effect but only a small additive effect was observed.

Conclusion A vaginal tablet offers an easy application and direct localized access to the cervix; adjacent to the cancerous tissue. The advantages of vaginal drug delivery include (i) bypassing hepatic first pass-effect; (ii) low systemic drug exposure; and (iii) higher bioavailability. A bilayer tablet provides an opportunity to deliver two active pharmaceutical ingredients (API) simultaneously for a synergistic pharmacological effect. Additionally, the different layers physically avoid chemical incompatibilities. Chitosan and polyacrylic acid are the polymers employed for their mucoadhesive property. These polymers also provide an extended and a controlled drug release rate. 5 -fluorouracil (5FU) a drug developed and used for the treatment of cancer for more than 50 years was selected as the primary API. Cell studies showed the first combination formulated; 5FU and disulfiram did not show a synergistic effect. Other API will be investigated in combination with $5 \mathrm{FU}$ in order to achieve the synergistic effect desired.

Disclosures The authors declare no conflict of interests.

\section{CHANGES IN THE PROTEOME OF CERVICOVAGINAL FLUID DURING HPV INFECTION IN HPV-VACCINATED WOMEN}

${ }^{1}$ Mariia lurova, ${ }^{2}$ Nataliia Starodubtseva, ${ }^{2}$ Kirill Gusakov, ${ }^{2}$ Niso Nazarova, ${ }^{3}$ Anna Bugrova, ${ }^{2}$ Alexandr Brzhozovzkiy, ${ }^{2}$ Vladimir Frankevich. ${ }^{1}$ Federal State Autonomous Educational Institution of Higher Education I.M. Sechenov First Moscow State Medical University of the Ministry of Health of the Russian Federation (Sechenov University); Federal State Budget Institution 'national Medical Research Center for Obstetrics, Gynecology and Perinatology Named after Academician V.I. Kulakov' Ministry of Health of Russia; ${ }^{2}$ Federal State Budget Institution 'national Medical Research Center for Obstetrics, Gynecology and Perinatology Named after Academician V.I. Kulakov' Ministry of Health of Russia; ${ }^{3}$ Federal State Budget Institution 'national Medical Research Center for Obstetrics, Gynecology and Perinatology Named after Academician V.I. Kulakov' Ministry of Health of Russia; N.M. Emanuel Institute of Biochemical Physics of Ras, Moscow, Russian Federation

\subsection{6/ijgc-2020-ESG0.32}

Methodology This study was approved by the Institute Research Medical Ethics Committee. The study involved 62 patients vaccinated against HPV, aged 19 to 45 years. The 